reduces the concentration of HDL cholesterol by transferring cholesteryl esters from HDL particles to lipoproteins. Consequently, inhibition of CETP might raise HDL-cholesterol levels and reduce the risk of atherosclerosis. To determine whether torcetrapib, a CETP inhibitor, could prevent the progression of carotid atherosclerosis in patients with mixed dyslipidemia, an international group of researchers conducted a randomized, double-blind trial involving 752 patients with mixed dyslipidemia, identified from 64 centers in North America and Europe.

Following an atorvastatin dose-titration run-in period, all patients remained on atorvastatin and were randomized to additionally receive either $60 \mathrm{mg}$ torcetrapib $(n=377)$ or placebo $(n=375)$ daily. Ultrasonography was used to assess carotid intima-media thickness at baseline and at 6,12 and 18 months after randomization. Mean duration of follow-up was 20 months.

Although, in comparison with baseline, torcetrapib significantly increased the HDLcholesterol concentration $(P<0.0001)$ and decreased the LDL-cholesterol concentration $(P<0.0001)$, the treatment did not substantially affect the rate of change in the maximum intima-media thickness; the rate of change was $0.025 \mathrm{~mm}$ per year in patients receiving torcetrapib plus atorvastatin and $0.030 \mathrm{~mm}$ per year in patients receiving atorvastatin plus placebo $(P=0.46)$. Moreover, systolic blood pressure increased significantly in those receiving torcetrapib compared with those given atorvastatin with placebo $(P<0.0001)$.

On the basis of the fact that torcetrapib has consistently failed to show any clinical benefit during trials, it will not be developed further.

Original article Bots ML et al. (2007) Torcetrapib and carotid intima-media thickness in mixed dyslipidaemia (RADIANCE 2 study): a randomised, double-blind trial. Lancet 370: 153-160

\section{Myocardial contrast echocardiography can accurately predict prognosis after AMI}

The risk of further cardiac events is high following acute myocardial infarction (AMI), especially after thrombolysis. Myocardial contrast echocardiography (MCE) is a relatively new technique that can determine infarct size by detecting contrast microbubbles within the myocardium at the capillary level. Dwivedi et al. determined whether residual myocardial viability as assessed by MCE could predict hard clinical events more accurately than could the clinical, electrocardiographic, biochemical and resting left-ventricular-function parameters currently widely used as prognostic markers.

The final analysis included 95 patients who underwent simultaneous transthoracic echocardiography and low-power MCE within $7 \pm 2$ days after experiencing AMl; 86 of these underwent thrombolysis. Over an approximately 4-year period there were eight cardiac deaths and seven nonfatal AMls. Elevated contrast defect index (CDI) on MCE-calculated by dividing the sum of the contrast intensity scores of all segments of the left ventricle by the number of evaluable segments - was the strongest univariate predictor of cardiac death, and of cardiac death or nonfatal AMI. Elevated $\mathrm{CDI}$ was also the strongest predictor of cardiac death on multivariate analysis $(P=0.01)$, and was the only parameter to independently predict death or nonfatal AMI $(P=0.002)$. Optimum CDI cutoff values correctly predicted survival, and survival or absence of recurrent AMI, in $99 \%$ and $95 \%$ of patients, respectively.

The authors believe that MCE could become the method of choice for assessing myocardial viability after AMI, but advise that their results require replication in larger multicenter studies before the technique can be widely recommended for use.

Original article Dwivedi G et al. (2007) Prognostic value of myocardial viability detected by myocardial contrast echocardiography early after acute myocardial infarction. J Am Coll Cardiol 50: 327-334

\section{Contractile reserve of the volume-overloaded ventricle is minimal}

The capacity for recovery of the right ventricle after surgical intervention for right ventricular to pulmonary artery conduit dysfunction is unclear. Previously, Coats et al. reported successful outcomes for the relief of pressure overload by nonsurgical restoration of the pulmonary valve. Now, to further understand postprocedural ventricular recovery, this group has examined the response of the right ventricle to the relief of volume overload by percutaneous pulmonary valve implantation (PPVI). 